incision will be the most judicious treatment. If the cyst be large, or there be a rapid secretion, $I$ should prefer a moderate incision, and the tent of lint.

The third case to which I would refer is one of very rare occurrence, indeed, I have not known it exactly described in our pathology. It is not the chronic abscess of Hunter; it is not, I believe, strumous, nor the cold abscess or lymph abscess of Chelius; nor the atheromatous, or the medullary sarcoma of Abernethy; nor the chronic or benign carbuncle of Sir Astley Cooper; nor is it anthracion, or malignant pustule. If I ventured to give a definition, I would term it "spongy carbuncle."

CASE 3.-Miss G-about forty years of age, of a very cheerful disposition, which she had preserved through life, although she had been afflicted with a variety of maladies, was attacked, three years since, by a severe hopatic disorder, attended by much complication. After nearly a year's sojourn in the country, she returned to London apparently convalescent. In a few weeks, however, a blush appeared between the superior angles of the scapulæ, attended by throbbing and tenderness. In a day or two this became of a deep hue, and doughy to the touch; still it was slow in its further progress. I did not imme. diately incise it, for I was not without hope that a combination of tonics and purgatives might induce a favourable change. Aromatic sulphuric acid and quinine were given, with an occasional aperient. In about a month, however, a slough formed and began to separate. Linseed poultices were applied, and a mild nitric acid lotion occasionally. The slough separated irregularly, adhering in its centre so very firmly, that I dissected the bulk of it carefully away, cutting through an insensible substance resembling very hard udder fat. The granulations became more healthy, and the cavity was rapidly healing, when a severe bilious attack again supervened. Directly, in place of healthy granulations, we had a second slough formed, surrounded by a pasty, languid sore. Tonics, with taraxacum and aloetics, were administered, with slight effect. The disease extended gradually deeper, forming a large sinus on the trapezius. The glazed, spongy mass rapidly increased, and although the tissues around it, especially the skin, were acutely painful, the fungus itself was completely void of sensation.

The mass was now so extensive, weighing probably about two pounds, that I was fearful of attempting its destruction by caustic or extirpation. I therefore introduced through the sinus and the spongy cushion, a thick skein of silk, by an eyed trochar and canula, with the hope of exciting another action and inducing absorption. The symptoms were in a day or two much amended, an unhealthy sanies, however, still flowing through both openings. In about two months an extensive absorption of the spongy mass was decided, and the abscess subsequently filled up with healthy granulation.

In about ten months from this period a red spot appeared on the top of the right shoulder. In about a fortnight I lanced it, discharging about an ounce of ichor. The probe introduced through this orifice rested above the scapnlar spine, on a thick, spongy, and perfectly insensible cushion, from which is now constantly flowing a dark, sanious fluid, the morbid mass having hitherto resisted our efforts for its destruction.

Storey's-gate, St. James's-park, January, 1848.

\section{CHLOROFORM IN MIDWIFERY.}

Bx W. M. FAIRBRoTHER, M.R.C.S.E., London.

As the question whether chloroform is useful or injurious in mid wifery can only be decided by facts, $I$ have subjoined the following cases :-

Mrs. W-, on the 12th of December, was attended by me during her labour. She had been for some months confined to her room, and was unable to walk without assistance; emaciated, irritable, and nervous. I was disposed to account for her inability to walk from the high position of the foetus in utero, the great distention of the abdomen, and the consequent strain upon the fascia lata of the thigh, and the cutaneous nerves which lie upon it. The dilatation of the os uteri commenced at about seven o'clock in the evening, and the head was found to present. The patient seemed to suffer most acutely while her inability to move increased. One drachm of chloroform was administered by means of a sponge, the vapour of which she readily inhaled. The pains continued regularly, but there was no expression of suffering. In half an hour the child was expelled, when she informed me, on her complete return to consciousness, that she was sensible of the presence of the pains, but the feeling of them was lost.
Coma in this case was not completely produced, and rapid recovery followed.

On the 21st of December I was called to attend Mrs. Sof Elliott's-row, St. George's-road; age about forty; labour natural. Chloroform was not used until within five minutes of the child's birth, and then with the intention of preventing that terrible feeling of distention which must accompany the passage of the head through the external opening. In this case the sponge was dropped, my hands being engaged, when, grasping it immediately, she inhaled the vapour with eagerness, and with manifest effect.

Mrs. F-, pregnant for the first time, was seized with labour-pains on the 22nd of December, at one o'clock P.M. Head presenting; os uteri gradually dilated. The pains increased in strength, and after many hours of suffering, she became impatient for the termination of labour. At one o'clock on the morning of the $23 \mathrm{rd}$, chloroform was administered, when the pains became less frequent, and, as far as could be judged by manual examination, diminished in intensity. There seemed, however, to be a re-action, and, shortly afterwards, the head of the child passed into the vagina. The effect of the first exhibition of chloroform having somewhat passed off, it was renewed. There was deficient secretion from the mucous follicles of the vagina. Cold cream was used, and the head with great difficulty cleared the os externum. Labour was concluded about half-past three, and during its last stage the patient seemed in a comfortable sleep, moaning slightly during the pains. It was not until during the extraction of the placenta that consciousness was partially restored, when Mrs. F — said, in ariswer to a question which was put, that the sensation of the removal of the after-birth was "as if a piece of paper had been taken off her." One ounce of chloroform was employed.

There can be no reasonable excuse for the non-administra. tion of chloroform, subject to certain rules. The patients who inhale it recover as rapidly as others; there is no aftersickness nor headach; but it ought never to be applied until the head is felt to be making some progress through the pelvis, except in cases of impaction, where it would be useful, as the pains became weaker, in procuring refreshing sleep and fresh energy. It is, however, a question whether secretion is not retarded if chloroform be used too soon, and there is a dry and unpliant vagina, which unguents do not much improve. Surely, however, the mere saving of agony to the mother will be a sufficient inducement to every practitioner to give a candid trial to the merits of chloroform.

London road, Southwark, December, 1847.

\section{PSEUDO-EPILEPTIC AFFECTION ARISING FROM DECAYED TEETH.}

Bx A. C. CASTLE, M.D., Surgeon-Dentist, New York. THE following singular and interesting case presented itself to my notice a short time since, and in itself offers abundant evidence (if any were required) of the necessity, not, as is contended, that a medical practitioner should receive a dentist's education, but that the dental student should receive a thorough education in all the branches of anatomy, physiology, \&c.

CASE.-A youth, about nineteen years of age, who had accompanied his mother to me, for dental advice, attracted my attention, in consequence of a peculiar idiotic expression of countenance, and dull, inanimate stare of the eye. I ventured to make some inquiries of the mother in reference to the lad, and she immediately entered into a history of his case. He had been a fine child, and grew up a smart, active boy, until about four years since, when his health had become maccountably impaired. He complained of almost continued headach, severe pains in the eyes, darting thence into the centre of the brain, which he described as resembling the sensation of "a hot spike, burning and pricking him." These symptoms were accompanied by a stiffiness of the cervical muscles, pain in deglutition, and a numbness in the right arm, together with pyrexia and a flushed countenance. About two years since he was first seized with general convulsions, which occurred every alternate day, sometimes, however, on the third day. These seizures were of varying degrees of violence and continuance; sometimes they were so mild as to present more the appearance of coma; at other times the contractions and rigidity of the muscular system were extreme. The fits always ended in vomiting, and were frequently followed by partial deafness. There was always great irritation of the nucous membrane of the nares, from which he was constantly pulling the hairs. 
The lad had been subjected to medical treatment in the hospitals of Dublin, and other cities in Ireland. A seton had been inserted in the nape of the neck, and an issue in the arm. Blisters, rubefacients, and a variety of topical remedies, had been employed, and all without any beneficial results. The mother at length despaired of his recovery, and had resigned herself to the conviction, that her son "must die an idiot."

Being engaged at the time, I requested the mother of the lad to bring him to me again, informing her that I desired to investigate his case, and that I entertained a vague suspicion, from the statement she had just made, that the case came within that class of diseases, "dento-neuralgia," in which the presence of decayed teeth produced a variety of alarming symptoms, indicating derangements of the nervous system, (a paper upon which the Editor did me the honour to publish in THE LANCET.*) She accordingly again called upon me, with her son, on the Ist of August, and informed me that on that very day he had had severe convulsions; indeed, he was still labouring under irritation of the subcutaneous muscular fibres. On examining him more attentively than on the occasion of his former visit, I perceived that the lad was of the sero-lymphatic temperament, and much attenuated in his form. I examined his mouth, and found that his teeth bore all the marks characteristic of his temperament; he had already "cut" the two inferior dentes sapientiæ, and the two upper ones were partially through the gums-an unusually early appearance of teeth which almost uniformly present themselves at a much later period. Their evidently imperfect organization had aided the rapid progress of decay, and the dentes sapientiæ were now little more than a crumbling mass. The molar teeth were all more or less decayed, and excessively tender. With the ready assent of the mother I extracted every decayed tooth, amounting in all to nine, including the dentes sapientiæ, each of the inferiors having at the extremities of the fangs an enormous abscess, comprised in a sac as large as a common field-bean. On two of the molar teeth similar abscesses of smaller size were discovered: these sacs contained a blackish, putrescent, fetid matter.

A few minutes after the operation the lad was seized with violent rigors. A small quantity of hot brandy-and-water revived him. I then prescribed a wash for the mouth, and five grains of the proto-iodide of mercury to be taken daily, with a saline purgative thrice a week, and liberal diet.

Sept. 14th.-Discontinued the use of medicines. Ordered a compound rhubarb pill to be taken occasionally. He has not had the slightest symptom of a return of the convulsive affec tion since the operation on his mouth. His health appears to be quite reëstablished; his appetite is good and his form begins to afford evidences of vigour, whilst his countenance, no longer clouded by an idiotic expression, has assumed an aspect of health and intelligence.

New York, Sept. 1847.

\section{CASE OF PARTIAL OCCIUSION OF VENA CAVA INFERIOR.}

Br W. J. LITTLE, M.D., PHXSICIAN TO THE LONDON HOSPITAL.

(Read before the Hunterian Society.)

Scirrho-encephaloid disease of the mesentery, mesenteric glands, heart, lungs, liver, and kidneys; also of the bronchial glands, and of the lymphatic glands of the neck, axilla, dc. +

Jorr S_-_-, aged fifty-two, admitted into the London Hospital, under Dr. Little, May 11th, 1847. States that he felt quite well until the 5th of April last, when he was attacked, whilst at work in the London Docks, with severe pains of the loins, and a feeling of excessive debility. This latter increased so much, that he was compelled to rest himself several times during his walk home. A week afterwards he was suddenly attacked with violent pains of the left side, extending from the last rib to the brim of the pelvis. These have continacd to the present time, being much augmented by lying on the affected side. About a fortnight since he notice? \& number of small, hard nodules, about the size of peas, on the left side of his abdomen, which have rapidly enlarged. They occasioned no pain unless he squeezed them. He presents the characteristic cacliectin appearance of malignant disease, and

I am indebted fro the cimical report of this case to the zeal and ability of J. W. Tripe, M.B. The reader may compare with it one of obliteration of vena cava supericr, recorded by myself in Tre LaNCET for August 28 th, $18 \div 7$. is reduced in flesh, as if suffering from affection of the mesenteric glands. A glance at the abdomen exhibits a large number of nodules, varying from one to three or four lines in diameter, distributed chiefly on the left side of the abdomen, and very hard, as if composed of scirrhous deposit. The greater number are situated immediately beneath the skin; one or two, however, appear to be deposited in the substance of the muscles. In addition to these nodules, one larger and more deeply seated is felt beneath the floating ribs of the left side. No tumour can be detected in the abdominal cavity. Percussion indicates that the liver extends from the lower border of the nipple to a finger's breadth below the margin of the last rib. The superficial veins of the abdomen and thorax are slightly congested and varicosed. The blood circulates through the abdominal veins in a retrograde courseviz., from below upwards. These veins, although varicosed, are not convoluted. The lower extremities are cedematous as high as the knee, and their superficial veins turgid and slightly enlarged. He has not had palpitation, cough, or dyspnœa; bowels are regular; urine small in quantity, lateritious, high-coloured, and passed with difficulty; pulse 90 , weak, soft, and regular; tongue furred and yellowish, with pale tip and edges; mucous membranes anæmic.

June 3rd.- Is much emaciated; the cutaneous tubercles have enlarged, some having attained the size of a filbert. The urine is still passed with difficulty; to-day normal in colour, quantity, and quality; it has not at any time contained blood. The external epigastric veins have latterly much increased in size, and are connected by large anastomosing veins with the superficial veins of the thorax. The transit of blood from below upwards, or from the system of the inferior cava into that of the superior cava, is very active. The veins of the spermatic cords, as far as can be detectcd by the touch, are not congested or varicosed. Disease of the heart or lungs cannot be detected; the heart's action, however, is somewhat feeble. The pain of the lumbar region has increased, and is situated on both sides of the vertebral column, just above its articulation with the pelvis; it is greater on the left than on the right side. Besides the tumours already deseribed, a number of others are now perceptible in the course of the lymphatic glands of the neck and axilla, and also of the deeper inguinal glands; there are also one or two on the back.

12th.-Complains much of the difficulty in micturition; his emaciation increases, and he bitterly laments that his strength is failing him fast; the pains have increased, and are occasionally lancinating, although usually dull, tensive, and gnawing. A distinct abdominal tumour is now manifest to the touch, extending from the umbilicus downwards several inches. He gradually sank, and died on the 15th.

From the evident obstruction to the course of the blood throagh the inferior cava, and from the absence of the varicosed condition of the spermatic veins, which seemed to indicate that the tumour compressed the cava below their point of entrance into it, it was surmised, previously to the detection of the abdominal tumour, that the patient had cancerous disease of the mesentery.

Post-mortem examination.-Body much emaciated; a large tumour felt through the abdominal parietes, deeply-seated, and apparently bound down to the spine. On clevating the abdominal muscles, a large quantity (about three pints) of bloody fluid (chiefly blood) was removed from the abdominal cavity, and also a large dark clot from around the lesser omentum, which was infiltrated with, and disorganized by, the effused blood. The source of the extravasation could not be detected; it was probably from a ruptured vein. The stomach was healthy, but its veins were distended with dark blood. The intestines, with the exception of the ilcum, (which contained a patch as large as a shilling, were tree from cancerous deposit; they were much contracted. The duodenum passed across, and was closely bound down to a large tumour, which extended from the lesser omentum to the upper part of the sacrum, overlapped the spinal column for a small distance on either side, and was fumly adherent to the anterior surfaces of all the lumbar vertbore except the first. The lesser omentum was infiltrated with the morbid growth; the liver contained numerous large masses (about the size of a small apple) of scirrho-encephaloid matter; it was somewhat enlarged, and weighed four ponds ten ounces. The pancreas and spleen were healthy. Both kidneys contained a few small scirvhous deposits, the largest being about the size of a small Spanish nut. The aorta was imbedded in the tumour, passing throngh it near to its ancerior surface, and divided, by a small process of maliguant disease, from the vena cava; it was diminished in size about one sixth; and a lerre quantity of 